\title{
Synthesis of Dendritic Silver Nanoparticles and Their Applications as SERS Substrates
}

\author{
Jinshan Yu and Xingui Zhou \\ Science and Technology on Advanced Ceramic Fibers and Composites Laboratory, College of Aerospace Science and Engineering, \\ National University of Defense Technology, Changsha 410073, China
}

Correspondence should be addressed to Jinshan Yu; jjss_y@163.com

Received 2 September 2013; Revised 7 November 2013; Accepted 28 November 2013

Academic Editor: Luís Cunha

Copyright (C) 2013 J. Yu and X. Zhou. This is an open access article distributed under the Creative Commons Attribution License, which permits unrestricted use, distribution, and reproduction in any medium, provided the original work is properly cited.

\begin{abstract}
The silver nanoparticles are synthesized by electrodeposition in ultradilute $\mathrm{Ag}^{+}$concentration electrolyte under high overpotential. The as prepared Ag nanoparticles, with the sizes ranging from 20 to $30 \mathrm{~nm}$, are arrayed orderly and formed dendritic morphology. The formation of this special dendritic nanoparticle structure can be contributed to the relatively high growth rate and the preferential growth directions along $\langle 111\rangle$ due to the high overpotential, as well as the relative small number of $\mathrm{Ag}^{+}$ions arriving at the $\mathrm{Ag}$ crystal surface per unit time due to the ultradilute $\mathrm{Ag}^{+}$concentration. Surface enhanced Raman scattering (SERS) experiments reveal that the as-prepared dendritic Ag nanoparticles possess high SERS properties and can be used as a candidate substrate for practical SERS applications to detect the Rhodamine $6 \mathrm{G}$ molecules.
\end{abstract}

\section{Introduction}

The surface enhanced raman scattering (SERS) is a technique that can determine an enhanced Raman signal when a Raman active-molecule is close to an appropriate metallic substrate surface [1]. Recently this technique has been developed into a valuable tool for chemical and biological sensing due to its high level of sensitivity and high spectroscopic precision [24]. Raman signal amplification depends on electromagnetic and chemical enhancement which arises from the interaction between molecules and the active substrate of metal nanostructures $[5,6]$. Previous studies have demonstrated that coinage metals, in particular, $\mathrm{Ag}$, usually provide much stronger SERS enhancements than alkali metals or transition metals because the surface-plasmon resonance of these freeelectron metals can be effectively excited by visible light [79]. Thus the nanostructured Ag are important candidates for practical SERS applications. Usually the unique physical and chemical properties of noble metal nanoparticles are highly dependent on their size, shape, and environment of the particles [10]. As a result, great attention has been directed toward the control of the noble metal nanoparticles size, size distribution, and their morphology. So far, many methods of preparing SERS-active Ag substrates have been extensively explored in order to obtain substrates with high enhancement ability and stability [11-13]. Here we report the newly synthesized arrayed dendritic Ag nanoparticles through a simple electrodeposited method and their application as a SERS substrate.

\section{Experimental Procedure}

The dendritic Ag nanoparticles were synthesized by electrochemical deposition method at room temperature. A classical three-electrode setup (Iviumstat electrochemical analyzer, Ivium Technology) was used to carry out the electrochemical experiments. All chemicals were of reagent grade and were used without further purification. Highly diluted $\mathrm{Ag}_{2} \mathrm{SO}_{4}$ was used to guarantee the nanostructure of the as-deposited products and the $\mathrm{pH}$ value of the plating solution was adjusted by adding $\mathrm{H}_{2} \mathrm{SO}_{4}$ to keep a suitable electrical conductivity. ITO glass sheets were used as the working electrodes on which the nanostructured $\mathrm{Ag}$ was deposited. A silver-silver chloride electrode was chosen as the reference and a pure Pt sheet positioned parallel to the ITO glass was used as the counter electrode. A salt bridge was used between the cell and the reference electrode. 


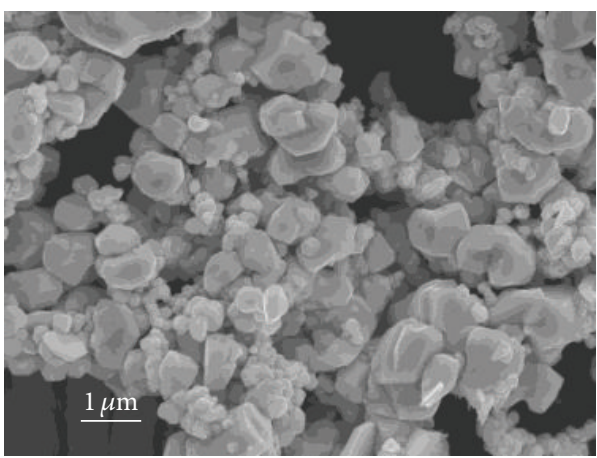

(a)

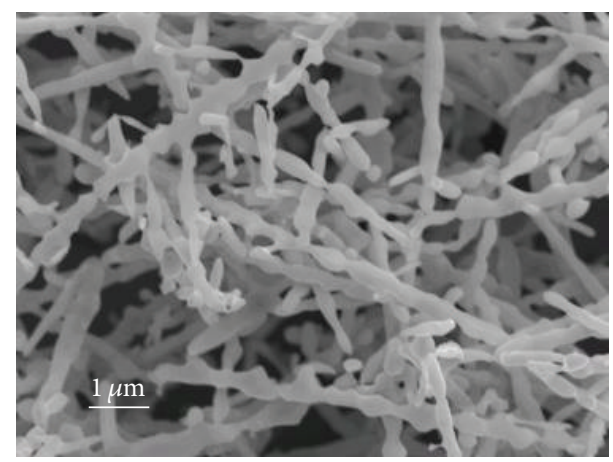

(b)

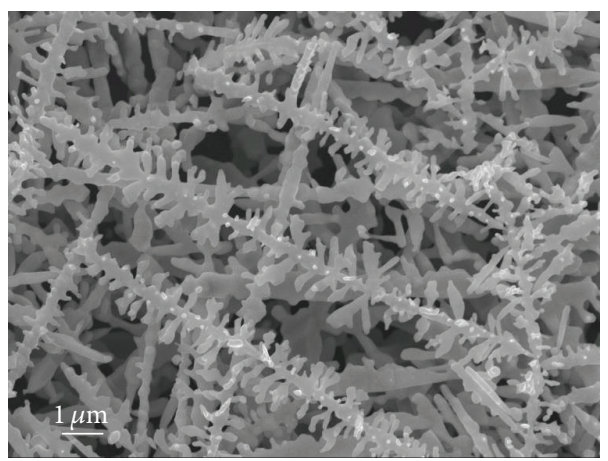

(c)

FIGURE 1: The SEM micrograph of Ag nanostructures deposited in $0.5 \mathrm{mM} \mathrm{Ag}_{2} \mathrm{SO}_{4}$ at different potentials: (a) $0 \mathrm{~V}$, (b) -0.1 V, and (c) -0.25 V.

The microstructure of the as-prepared nanostructured Ag was inspected by a Hitachi S-4800 scanning electron microscope (SEM) equipped with an X-ray energy dispersive spectroscopy (EDX). Surface enhanced Raman scattering was measured from 400 to $1700 \mathrm{~cm}^{-1}$ at room temperature by a Brucher VERTEK 70 Raman spectrometer with a $514.5 \mathrm{~nm}$ $\mathrm{Ar}$ ion laser. The laser power focused on the sample is $50 \mathrm{~mW}$ and the acquisition time is 1 second. The Rhodamine 6G (R6G), which was used as probe molecule, was dissolved into pure water $(18.2 \mathrm{M} \Omega)$. Before SERS measurements, the ITO glasses deposited with the dendritic Ag nanoparticles were immersed in the solution for $2 \mathrm{~h}$ for sufficient molecule adsorption and then were dried in air for 4 hours.

\section{Results and Discussion}

As we had reported early, the noble metal nanostructures can be fabricated by electrodeposition method in a highly diluted solution with a special deposition mechanism [14]. The shape of the as-deposited nanostructured noble metals can be controlled by tuning the depositing potentials. In this study we firstly studied the electrodeposition of Ag nanostructures at different potentials in a mixed electrolyte composed of $0.5 \mathrm{mM} \mathrm{Ag}_{2} \mathrm{SO}_{4}$ and $1 \mathrm{M} \mathrm{H}_{2} \mathrm{SO}_{4}$ in nanopure deionized water. Figure 1 shows the morphology of Ag nanostructures deposited at different potentials. As shown in Figure 1(a), at deposition potentials of $0 \mathrm{~V}$, the Ag particles are formed with the size of $\sim 1 \mu \mathrm{m}$. With more negative potential $(-0.1 \mathrm{~V})$, the bamboo-like Ag rods with the diameter of $\sim 300 \mathrm{~nm}$ are formed, as shown in Figure 1(b), which implies the preferred growth of the Ag crystals. At an overpotential of $-0.25 \mathrm{~V}$, dendritic Ag nanostructures with size scale of $\sim 200 \mathrm{~nm}$ are obtained, as shown in Figure 1(c).

Then the Ag nanostructures are electrochemically deposited in more dilute solution. The concentration of $\mathrm{Ag}_{2} \mathrm{SO}_{4}$ decreases to $0.2 \mathrm{mM}$. Figure 2 shows the SEM micrograph of Ag nanostructure electrochemically deposited at the overpotential of $-0.25 \mathrm{~V}$. The feather-like Ag dendritic nanostructure is obtained, as shown in Figure 2(a). The EDX result (Figure 2(b)) confirms that the as-obtained Ag nanostructure is only composed of Ag. High-magnification SEM micrograph (Figure 2(c)) shows that a single Ag dendrite consists of many subdendrites. Figure 2(d) shows an ultra-high-magnification SEM micrograph of the $\mathrm{Ag}$ sub-dendrite. It clearly shows that an $\mathrm{Ag}$ subdendrite is composed of $\mathrm{Ag}$ nanoparticles with the size of $\sim 30 \mathrm{~nm}$, which is arrayed into a dendritic morphology.

The formation of this special dendritic nanoparticle structure can be contributed to the electrochemical deposition mechanism in ultradilute solution. At the deposition potential of $-0.25 \mathrm{~V}$, the overpotential is much negative than the standard electrode potential of $\mathrm{Ag}(+0.799 \mathrm{~V})$. At this deposition potential the Ag crystals should have a relatively high growth rate and the preferential growth direction along $\langle 111\rangle$ [14]. However, the $\mathrm{Ag}^{+}$in electrolyte are very dilute; thus the number of $\mathrm{Ag}+$ ions arriving at the $\mathrm{Ag}$ crystal surface per unit time is small. The arrived $\mathrm{Ag}^{+}$have enough time to find the coordination sites to decrease the surface energy. 


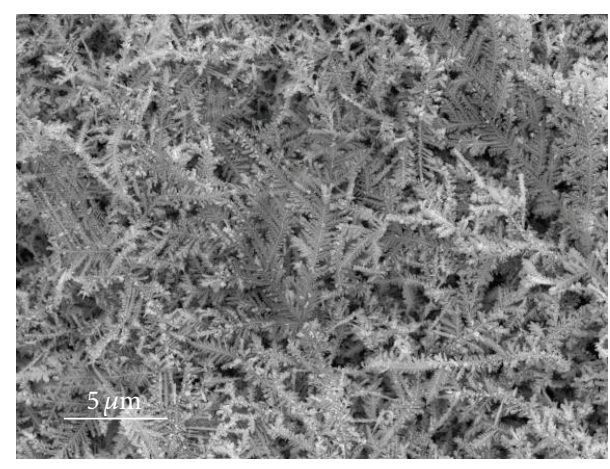

(a)

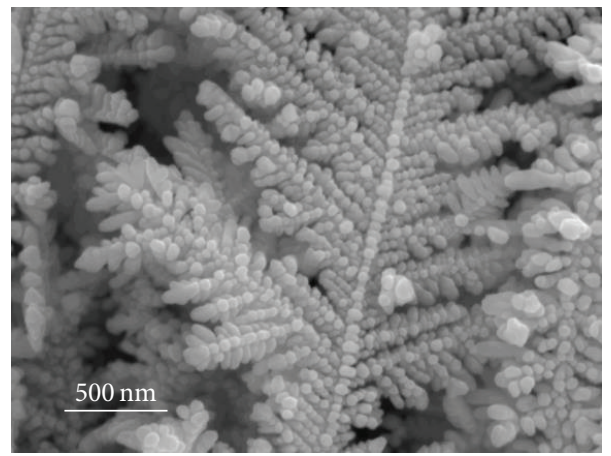

(c)

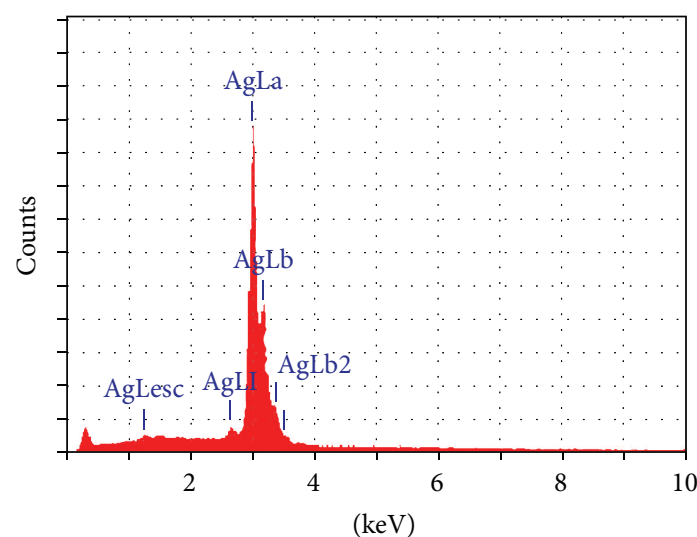

(b)

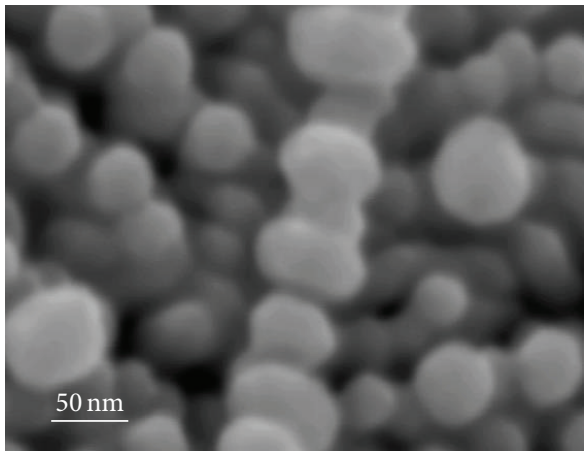

(d)

FIGURE 2: The SEM micrograph of Ag nanostructure electrochemically deposited in $0.2 \mathrm{mM} \mathrm{Ag}_{2} \mathrm{SO}_{4}$ with the overpotential of -0.25 V: (a) Low magnification, (b) EDX result, (c) high magnification, and (d) ultra-high magnification.

Thus the spherical Ag nanoparticles are formed. It can be concluded that the arrayed dendritic nanoparticle structure is formed due to the high overpotential and the ultradilute $\mathrm{Ag}^{+}$concentration.

Figure 3 shows the SERS spectrum of R6G molecules on dendritic Ag nanoparticles. The concentration of R6G solution is $10^{-10} \mathrm{M}$. It can be seen that the SERS intensity is relatively strong, which implies that these dendritic $\mathrm{Ag}$ nanoparticles have the potential to detect more dilute R6G solution. It is generally believed that SERS is a highly heterogeneous process due to local enhancements at certain "hot spots" coming from the nanostructure [15]. In this dendritic Ag nanoparticle, the positions of each Ag nanoparticle are ordered and relative fixed; therefore the aggregations of $\mathrm{Ag}$ nanoparticles cannot occur. These arrayed Ag nanoparticles act as the "hot spots", trapping the analyte in those junctions, leading to a high SERS activity. It confirms that the asprepared dendritic Ag nanoparticles can be used as a high performance substrate for practical SERS applications to detect the R6G molecules.

\section{Conclusion}

In summary, the silver nanoparticles are synthesized by electrodeposition in ultradilute $\mathrm{Ag}^{+}$concentration electrolyte

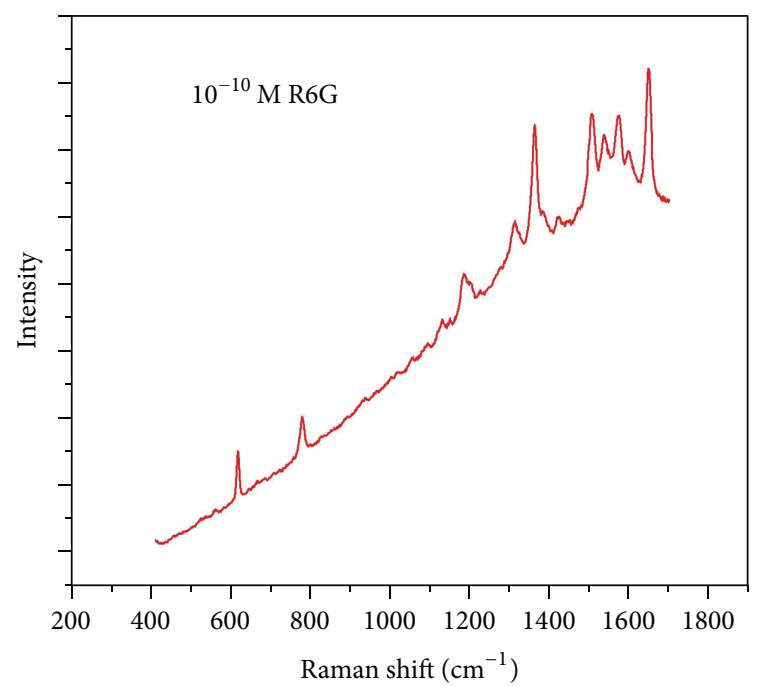

FIGURE 3: The SERS spectrum of R6G molecules on dendritic Ag nanoparticles.

under high overpotential. The as-prepared dendritic Ag nanoparticles possess high SERS properties and can be used as a candidate substrate for practical SERS applications to detect the Rhodamine $6 \mathrm{G}$ molecules. 


\section{Acknowledgments}

This work was financially supported by the Fundamental Research Fund of National University of Defense Technology, the Research Fund of State Key Laboratory of CFC (Grant no. 9140C820301110C8201), and the National Natural Science Foundation of China (Grant no. 51372274).

\section{References}

[1] C. L. Haynes, A. D. McFarland, and R. P. van Duyne, "Surfaceenhanced: Raman spectroscopy," Analytical Chemistry, vol. 77, no. 17, pp. 338-346, 2005.

[2] K. Kneipp, R. F. Aroca, H. Kneipp, and E. Wentrup-Byrne, New Approaches in Biomedical Spectroscopy, Oxford University Press, Oxford, UK, 2007.

[3] F. C. Adam, K. Khashayar, T. Shi-Yang et al., "In situ SERS probing of nano-silver coated individual yeast cells," Biosensors and Bioelectronics, vol. 49, pp. 536-541, 2010.

[4] W. Wu, M. Wu, Z. Sun et al., "Morphology controllable synthesis of silver nanoparticles: optical properties study and SERS application," Journal of Alloys and Compounds, vol. 579, pp. 117123, 2013.

[5] M. Moskovits, "Surface-enhanced spectroscopy", Reviews of Modern Physics, vol. 57, no. 3, pp. 783-825, 1985.

[6] A. Campion and P. Kambhampati, "Surface-enhanced Raman scattering," Chemical Society Reviews, vol. 27, no. 4, pp. 241-250, 1998.

[7] Z. Gan, A. Zhao, M. Zhang et al., "A facile strategy for obtaining fresh Ag as SERS active substrates," Journal of Colloid and Interface Science, vol. 366, no. 1, pp. 23-27, 2012.

[8] Z. Q. Tian, B. Ren, and D. Y. Wu, "Surface-enhanced Raman scattering: from noble to transition metals and from rough surfaces to ordered nanostructures," The Journal of Physical Chemistry B, vol. 106, no. 37, pp. 9463-9483, 2002.

[9] B. K. Jena and C. R. Raj, "Seedless, surfactantless room temperature synthesis of single crystalline fluorescent gold nanoflowers with pronounced SERS and electrocatalytic activity," Chemistry of Materials, vol. 20, no. 11, pp. 3546-3548, 2008.

[10] C. Burda, X. Chen, R. Narayanan, and M. A. El-Sayed, "Chemistry and properties of nanocrystals of different shapes," Chemical Reviews, vol. 105, no. 4, pp. 1025-1102, 2005.

[11] L. Y. Chen, Y. S. Yu, T. Fujita, and M. W. Chen, "Nanoporous copper with tunable nanoporosity for SERS applications," Advanced Functional Materials, vol. 19, no. 8, pp. 1221-1226, 2009.

[12] J. Fu, Z. Cao, and L. Yobas, "Localized oblique-angle deposition: Ag nanorods on microstructured surfaces and their SERS characteristics," Nanotechnology, vol. 22, no. 50, Article ID 505302, 2011.

[13] X. Hou, X. Zhang, S. Chen et al., "Facile synthesis of SERS active Ag nanoparticles in the presence of tri-n-octylphosphine sulfide," Applied Surface Science, vol. 257, no. 11, pp. 4935-4940, 2011.

[14] J. Yu, T. Fujita, A. Inoue, T. Sakurai, and M. Chen, "Electrochemical synthesis of palladium nanostructures with controllable morphology," Nanotechnology, vol. 21, no. 8, Article ID 085601, 2010.

[15] S. J. Lee, A. R. Morrill, and M. Moskovits, "Hot spots in silver nanowire bundles for surface-enhanced Raman spectroscopy,"
Journal of the American Chemical Society, vol. 128, no. 7, pp. 2200-2201, 2006. 

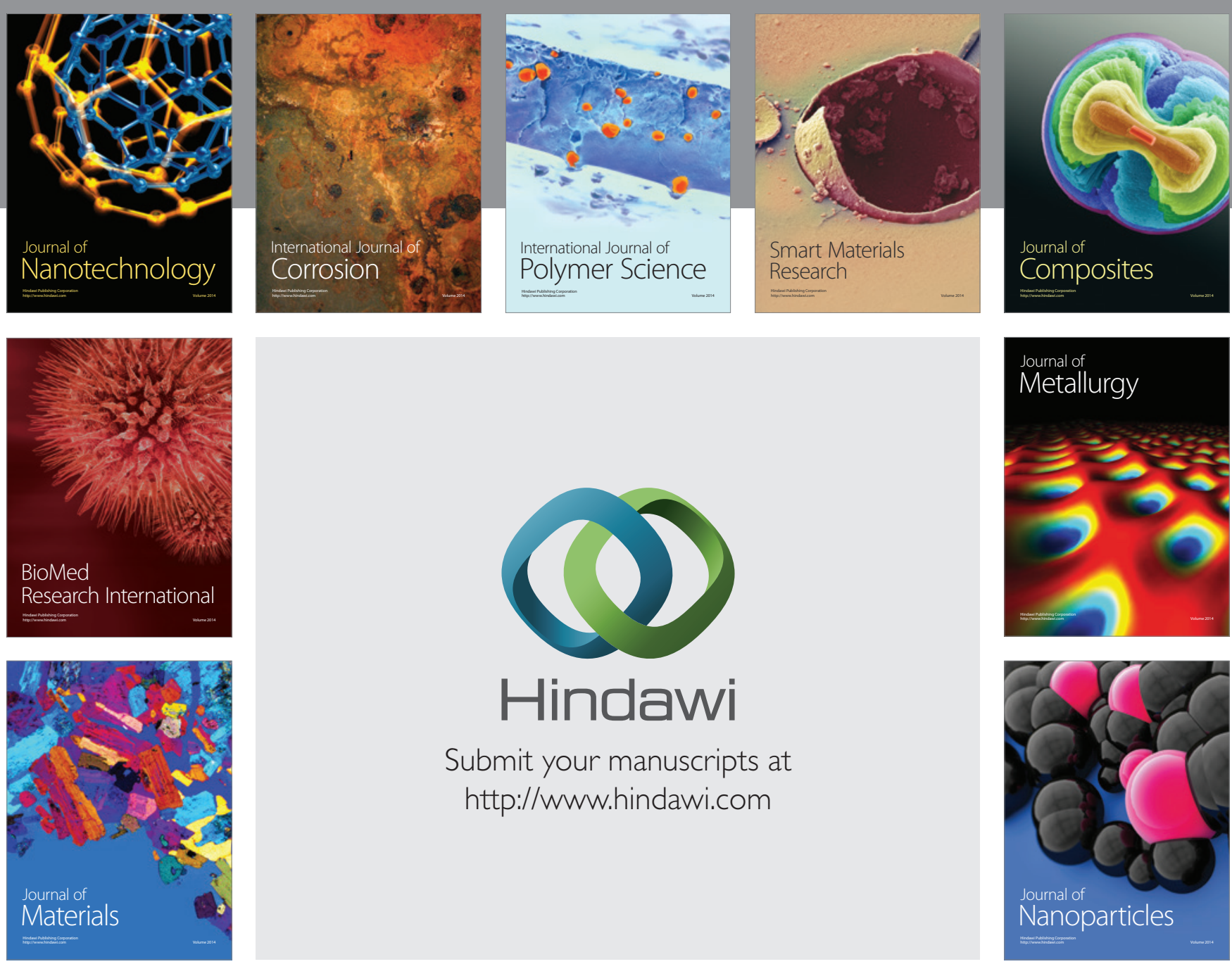

Submit your manuscripts at http://www.hindawi.com
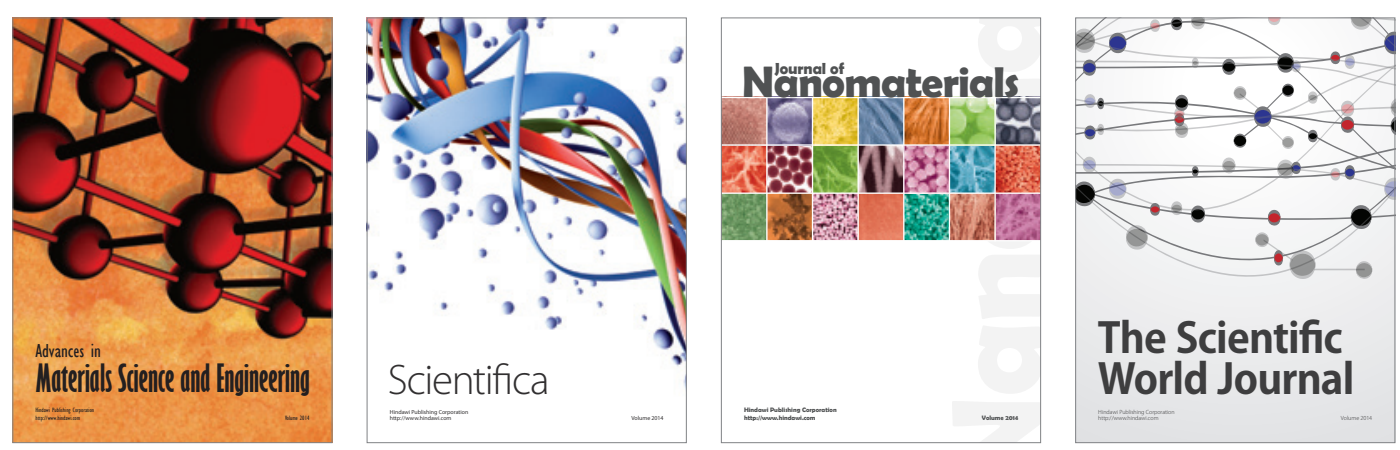

\section{The Scientific World Journal}
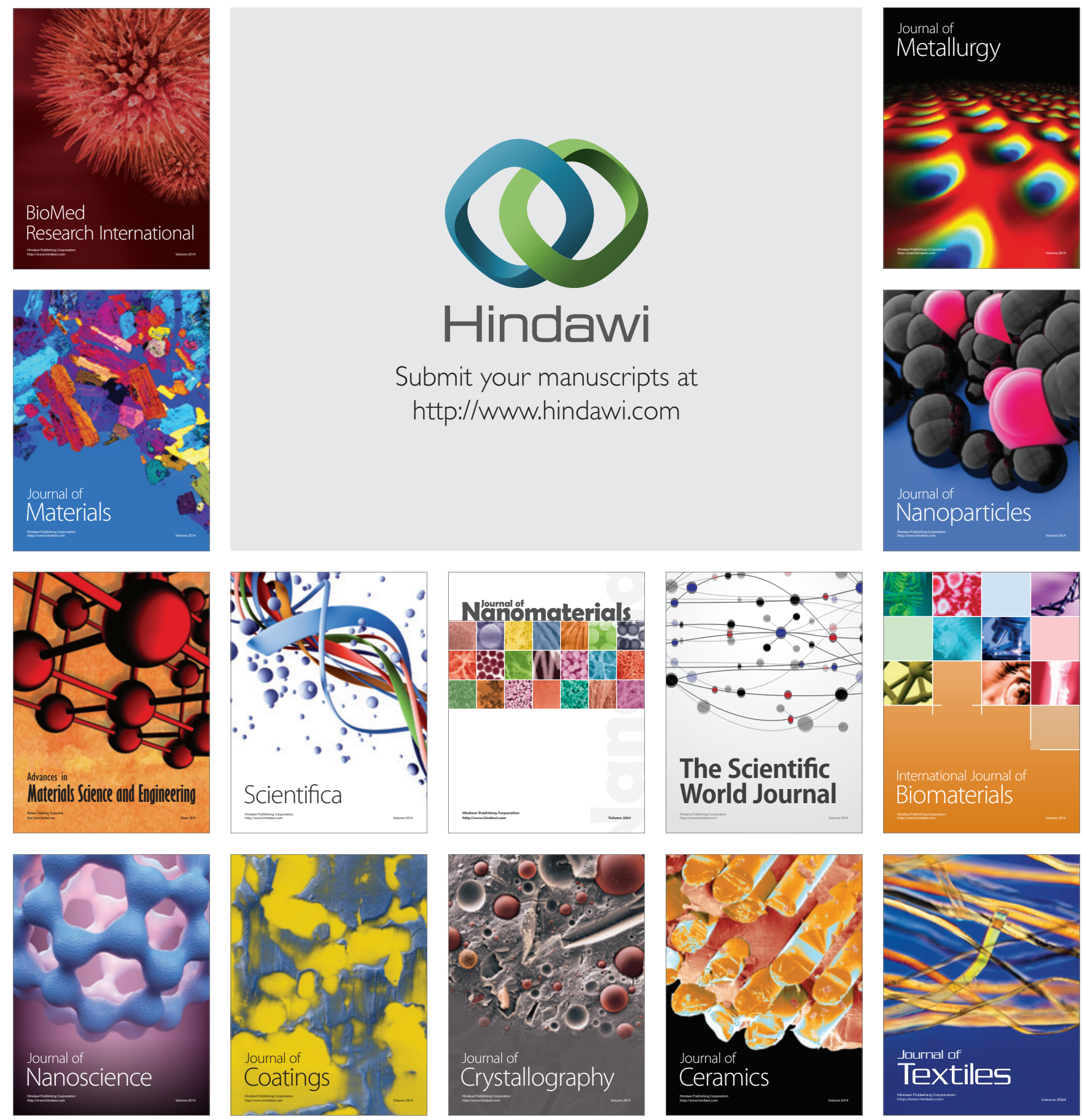